\title{
Brain Levels of Reduced Glutathione and Malondialdehyde in Honey-Fed Wistar Rats
}

\author{
Prosper Ejiro Awhin ${ }^{1 *}$, Ugochukwu Enyinnaya Uzuegbu, \\ Evelyn Ojugbeli ${ }^{1}$ and Lily Oghenevovwero Otomewo ${ }^{2}$
}

\author{
${ }^{1}$ Department of Medical Biochemistry, Delta State University, Abraka, Delta State, Nigeria. \\ ${ }^{2}$ Department of Pharmacology and Therapeutics, Delta State University, Abraka, Nigeria. \\ http://dx.doi.org/10.13005/bbra/2936
}

(Received: 26 August 2021; accepted: 23 October 2021)

\begin{abstract}
This research sought to verify the effect of natural honey on brain levels of malondialdehyde (MDA) and reduced glutathione (GSH) in rats. Forty nine male and female Wistar rats were used for the experiment. The rats were allotted into seven groups of seven rats in each group. For one month, rats in groups $1-4$ were fed with $100 \%$ feed, $20 \%, 30 \%$ and $40 \%$ honey respectively. The remaining 3 groups were fed with amounts of refined fructose and glucose equivalent to those in $20 \%, 30 \%$ and $40 \%$ honey. The brains were then excised, homogenized and used for biochemical analysis. Results showed that honey in all concentrations caused a significant increase in GSH levels but only $20 \%$ honey caused a significant decrease in MDA level when compared with control. Also, fructose feeding at $20 \%, 30 \%$ and $40 \%$ increased both brain GSH and MDA levels. Consequently, the influence of GSH as an antioxidant against brain lipid peroxidation needs further studies for better understanding since an increase in GSH for fructose- and honey-fed rats did not cause a simultaneous decrease in MDA content.
\end{abstract}

Keywords: Brain level; Glutathione; Honey-fed Wistar rats; Malondialdehyde.

Honey is a natural alternative to plain sugar. It is a sweet gold-coloured viscous substance which originates from the nectars of honey bees ${ }^{1}$. The constituents of honey vary and mainly depend on the source. Honey contains at least 181 substances. It is also a super saturated solution of compound sugars; fructose (38\%) and glucose (31\%). It also contains proteins, minerals, enzymes and vitamins ${ }^{2,3}$.

Honey holds numerous medicinal and health benefits as a natural food supplement. These benefits of honey have been reported to be related to its antioxidant, antibacterial ${ }^{4}$, antifungal ${ }^{5}$, antiviral ${ }^{6}$, antitumor $^{7}$, anti-inflammatory ${ }^{8}$, antidiabetic ${ }^{9}$, immunomodulatory ${ }^{10}$, etc. ability. Existing literary evidence suggests that honey is capable of protecting different body organs such as the brain, liver and kidneys from damage by acting as an antioxidant or enhancing cellular antioxidant defence systems ${ }^{11-13}$. It has also been demonstrated that honey plays a significant role in attenuating oxidative stress-induced cell death ${ }^{14}$. Furthermore, previous research has shown that $1.2 \mathrm{~g} / \mathrm{kg}$ of honey caused an elevation in level and activity of antioxidant agents in healthy humans ${ }^{15}$.

Oxidative stress has been posited to be a direct consequence of an imbalance between free radical species and antioxidants which results in 
debilitating effects in various organs of the body. It usually results either when the free radical species (e.g. malondialdehyde; MDA) activity is enhanced or when antioxidant agents such as reduced glutathione (GSH), superoxide dismutase (SOD) or catalase (CAT) are depleted ${ }^{1}$.

Typically, the brain is believed to be the organ most vulnerable to oxidative stress. Therefore, this study attempts to ascertain the significant changes (if any) in GSH and MDA levels in the brains of Wistar rats fed with varying amounts of natural honey.

\section{MATERIALS AND METHODS}

\section{Experimental animals}

Forty nine (49) Wistar rats weighing 61$111 \mathrm{~g}$ used in the present research were obtained from the Animal House, Faculty of Basic Medical Sciences, Delta State University, Abraka, Delta State. They had unrestricted access tofood (i.e. growers' mash obtained from Top-Feeds, Sapele, Delta State, Nigeria) and water. The animals were subsequently housed in metal cages under appropriate conditions of $12 \mathrm{~h}$ light: $12 \mathrm{~h}$ dark cycle. Drugs

Honey was purchased from Ewu Catholic Monastery, Edo State, Nigeria. Glucose, fructose (Food Grade) and sodium chloride (AnalaR Grade) were obtained from BDH Chemicals Limited, Poole, England.

\section{Chemicals}

All reagents and test kits used in this research were purchased from TECO Diagnostics, Anaheim CA, USA.

\section{Experimental design}

The rats were sorted and arranged into seven groups labelled 1-7. Each group contained seven rats $(n=7)$. During grouping, the seven groups were sorted and weighed so that the mean weights of all groups were approximately the same. The mean weights and standard deviations of each rat were then recorded accordingly. At the end of every week, the mean weights and standard deviations were recorded for each group and this was done for four weeks.

The rats were either fed on growers' mash that was honey- or sugar-free (control group) or contained 20\% (Group 2), 30\% (Group 3), 40\% (Group 4) honey or fructose/glucose equivalent to amounts in 20\% (Group 5), 30\% (Group 6) and $40 \%$ (Group 7) honey for four weeks. The key players in the research are linked and illustrated in Figure 1.

\section{Preparation of brain tissue homogenate}

Upon completion of the feeding periods, the overnight-fasted animals were then sacrificed by euthanization and their respective brains were harvested and collected into well-labelled tubes for the biochemical analysis. One gram $(1 \mathrm{~g})$ of wet brain tissue was homogenized in $9.0 \mathrm{~mL}$ of normal saline and the resultant supernatant was kept frozen until analysis ${ }^{16}$.

\section{Biochemical investigation}

The biochemical parameters measured include the levels of malondialdehyde (MDA) and reduced glutathione (GSH).

Brain reduced glutathione (GSH) was measured by the Ellman method ${ }^{16}$. On the other hand, lipid peroxidation in the brain was estimated by Thiol-Barbituric Acid Reactive Species (TBARS) using malondialdehyde (MDA) as standard ${ }^{17}$.

\section{Statistical analysis}

The Mean \pm Standard Deviation (SD) of the groups was calculated and values were expressed as such. Significant difference between means was evaluated by analysis of variance (ANOVA). Post-test analysis was done using Tukey's post-hoc test. Values of $\mathrm{p}<0.05$ were considered as statistically significant.

\section{RESULTS AND DISCUSSION}

Honey is a natural viscous sweetener

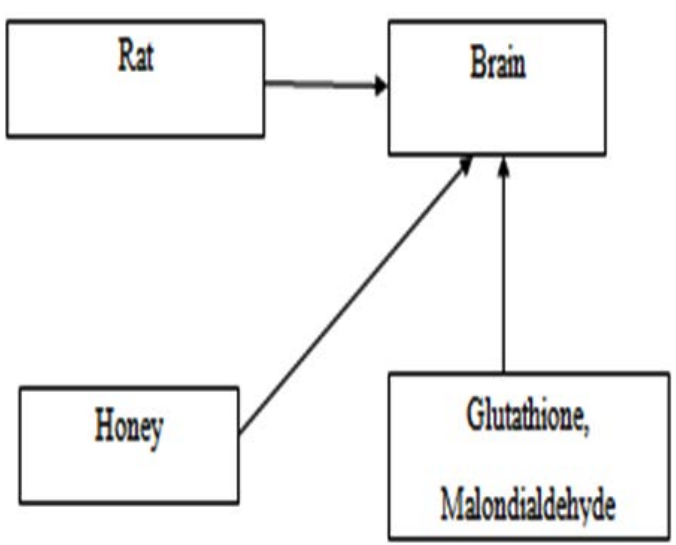

Fig. 1. Major factors in the experiment 
commonly used as food and in medical products such as drugs and ointments ${ }^{1}$. The present study suggests that honey possesses neuroprotective effect via the enhancement of antioxidant activity. The results of the present study revealed that honey feeding $(20 \%, 30 \%$ and $40 \%)$ significantly $(\mathrm{p}<0.05)$ increased brain GSH in a pattern that depends on the amount. However, only the higher doses of fructose/glucose feeding induced significant $(p<0.05)$ increase in brain GSH levels. On the other hand, changes in brain MDA levels for both honey and fructose-fed groups did not differ significantly ( $>0.05$ ) from the values in the control group. An exception was observed in the fructose group with $40 \%$ honey equivalent which resulted in a significant $(p<0.05)$ increase in MDA levels (Figure 1). This suggests that MDA levels progressively increased as honey equivalents increased in the fructose groups. This observed pattern was similar to the trend observed for GSH (Figure 2).

The results from the present study reveal that $20 \%$ honey caused a decrease in brain levels of GSH and MDA while 30 and $40 \%$ caused an increase in MDA and GSH. Honey is a potential natural antioxidant that works by enhancing the level and activity of endogenous antioxidants such as reduced glutathione (GSH) in a pattern that seems dose-dependent. This is in line with previous studies which suggested that treatment of animals with honey improved the brain antioxidant status (measured by catalase, GSH and superoxide dismutase) but no significant effect was observed on MDA level, which means honey acts by possibly increasing the antioxidant capacity needed to abrogate the effect of oxidative stress induced by lead exposure ${ }^{1,18}$. Also in the present study, the enhancing effect of honey on GSH level was observed in groups fed with fructose/glucose equivalent to honey amounts. Also, the results from this study is backed up by a previous study which showed that honey acted as an antioxidant by drastically reducing the index of hypertension and enhancing antioxidant activities (measured by GSH, GSSH and GPx) in diabetic spontaneous hypertensive rats ${ }^{12}$.

Furthermore, malondialdehyde (MDA) concentration was determined in the present study. MDA is considered a potent biomarker of lipid peroxidation which is known to be a key mechanism of oxidative stress formation ${ }^{3}$. Therefore, any substance capable of depleting MDA is considered a free radical scavenger.

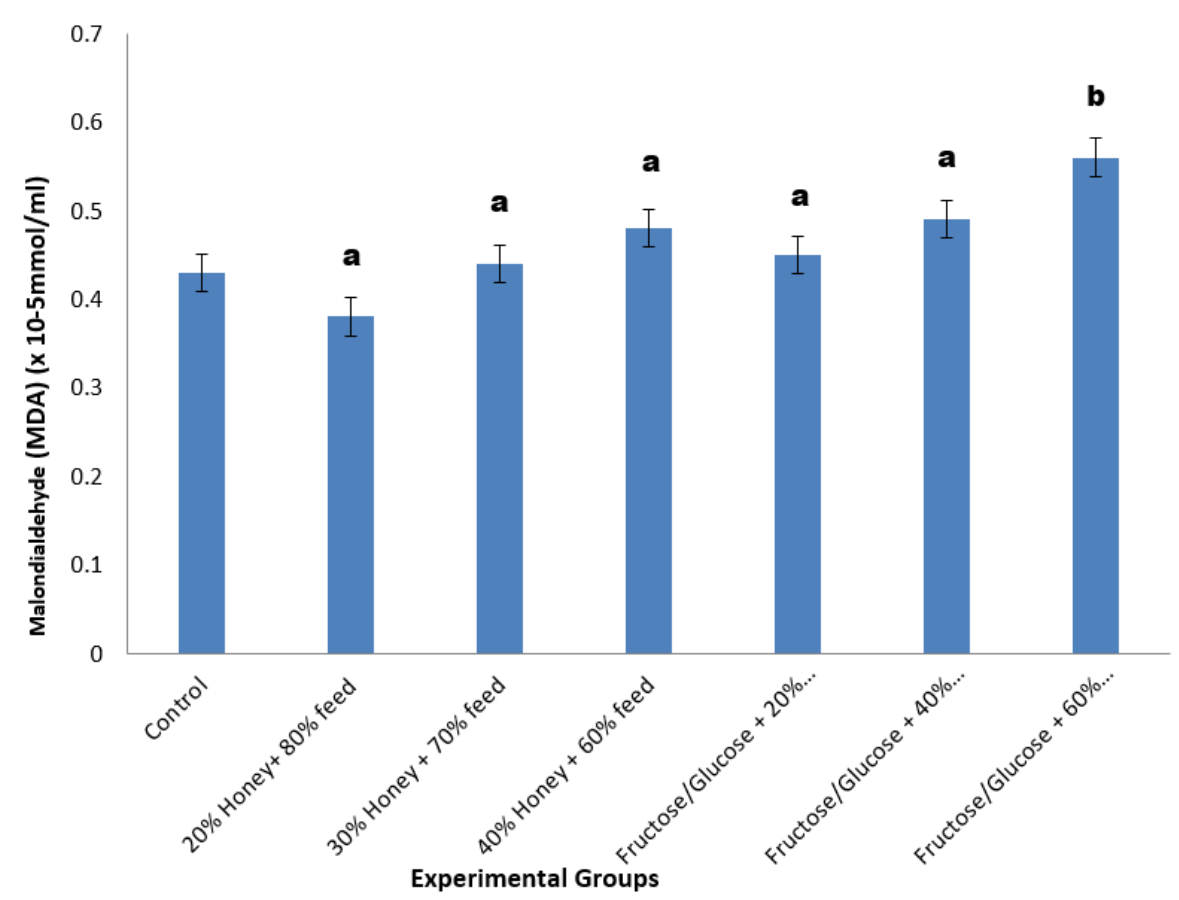

Fig. 2. Brain malondialdehyde (MDA) levels induced by honey feeding in Wistar rats 


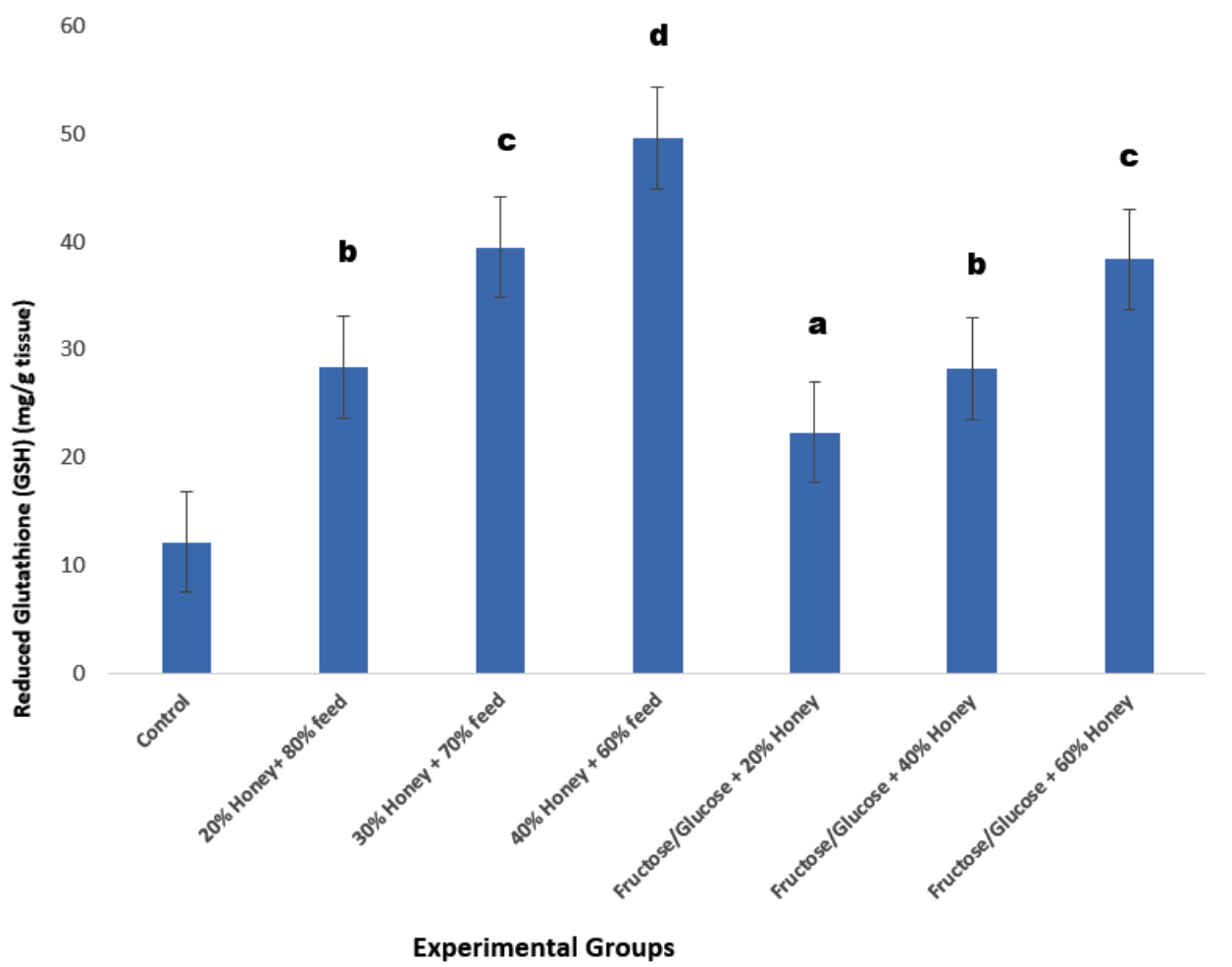

Values are expressed as Mean $\_$SD ( $=7$ rats/group). Values that bear different letter superscripts on a given column differ significantly $(\mathrm{p}<0.05)$

Group 1: Treated as control (100\% feed)

Group 2: Treated with $20 \%$ honey, $80 \%$ feed

Group 3: Treated with $30 \%$ honey, $70 \%$ feed

Group 4: Treated with $40 \%$ honey, $60 \%$ feed

Group 5: Treated with fructose/glucose equivalent to amounts in $20 \%$ honey

Group 6: Treated with fructose/glucose equivalent to amounts in $30 \%$ honey

Group 7: Treated with fructose/glucose equivalent to amounts in $40 \%$ honey

Fig. 3. Reduced glutathione (GSH) levels induced by honey feeding in Wistar rats

However, the present study shows that honey did not significantly reduce the brain MDA content when compared with the control group ${ }^{1}$ but the minimal amounts of fructose did (i.e. $20 \%$ and $30 \%)^{19}$. Although this experiment suggests that a high-fructose diet (i.e. $40 \%$ ) induced oxidative stress by significantly increasing MDA levels ${ }^{20}$, the present study agrees with previous reports of the oxidative stress-inducing ability of dietary fructose via a mechanism linked to increasing MDA level ${ }^{21}$.

\section{CONCLUSION}

From the available evidence in the present study, brain of rats fed with honey showed significantly increased GSH levels and non-significantly increased MDA levels as doses increased. This implies that the enhanced antioxidant activity of honey is still associated with a measure of increased levels of prooxidants. The results from this experiment therefore furnish industries with valuable information on the suitable concentrations of honey assists in determining the concentration of honey that can be added to food and medical products. Also, from the foregoing discussion, the antioxidant enhancement effect of honey may be unsubstantial in potentially preventing brain lipid peroxidation induced by either honey or fructose feeding in rats. Therefore, the mechanism by which honey attenuates lipid 
peroxidation and possibly reverses oxidative stress in brain tissue needs further studies.

\section{ACKNOWLEDGEMENT}

I am most grateful to Obitaba-Eraguonona Suvwe for being instrumental during the process of data collection and for providing useful scientific information that improved the quality of this research report. I also acknowledge the technical support of the laboratory staff members of the Department of Medical Biochemistry, Delta State University, Abraka, Nigeria. The advisory role of Dr. I. Onyesom is also highly appreciated.

\section{Conflict of Interest}

The authors declare no conflicts of interest.

\section{Funding Sources}

support.

This research received no external funding

\section{REFERENCES}

1. Abdulmajeed W. I, Suleiman H. B, Zubayr M. O, Imam A, Amin A, Biliaminu S. A. et al. Honey prevents neurobehavioural deficit and oxidative stress induced by lead acetate exposure in male Wistar rats- a preliminary study. Metab Brain Dis, 2015: 8pages

2. Rahman K. Phytochemical analysis and chemical compositionof different branded and unbranded honey samples. International Journal of Microbiological Research, 2013; 4(2): 132-137

3. Ahmed S, Sulaiman S. A, Baig A. A, Ibrahim M, Liaqat S, Fatima S, et al. Honey as a potential natural antioxidant medicine: an insight into its molecular mechanisms of action. Oxidative Medicine and Cellular Longevity, 2018; 19 pages.

4. Sherlock O, Dolan A,Athman R,et al.Comparison of the antimicrobial activity of Ulmo honey from Chile andManuka honey against methicillinresistant Staphylococcus aureus, Escherichia coli and Pseudomonas aeruginosa. BMC Complementary and Alternative Medicine, 2010; 10(1): 47.

5. Irish J, Carter D. A, Shokohi T, and Blair S. E. Honey has an antifungal effect against Candida species. Medical Mycology, 2006; 44(3): 289-29.

6. Shahzad A. and Cohrs R. J. In vitro antiviral activity of honey against varicella zoster virus (VZV): a translational medicine study for potential remedy for shingles. Translational Biomedicine, 2012; 3(2): 2.

7. Othman N.H. Does honey have the characteristics of natural cancer vaccine? Journal of Traditional and Complementary Medicine, 2012; 2(4): 276-283.

8. Molan P. Manuka Honey as a Medicine, Global Bioactive Summit, Hamilton, 2001.

9. Erejuwa O. O, Gurtu S, Sulaiman S. A. Ab Wahab M. S, Sirajudeen K. N, and Salleh M. S. Hypoglycemic and antioxidant effects of honey supplementation in streptozotocininduced diabetic rats. International Journal for Vitamin and Nutrition Research, 2010; 80(1): 74-82.

10. Morariu I. D, Schiriac E. C, Ungureanu $\mathrm{D}$, and Cuciureanu R. Immune response in rats following administration of honey with sulfonamides residues. Revista Român ã de Medicin ã de Laborator, 2012; 20(1): 63-72.

11. Alvarez-Suarez J. M, Giampieri F, Cordero M, et al. Activation of AMPK/Nrf2 signalling by Manuka honey protects human dermal fibroblasts against oxidative damage by improving antioxidant response and mitochondrial function promoting wound healing. Journal of Functional Foods, 2016; 25: 38-49

12. Erejuwa O. O, Sulaiman S. A,Wahab M. S. A, Sirajudeen K. N. S, Salleh M. S. M. and Gurtu S. Differential responses to blood pressure and oxidative stress in streptozotocin-induced diabetic wistar-Kyoto rats and spontaneously hypertensive rats: effects of antioxidant (honey) treatment. Int J Mol Sci, 2011; 12(3): 1888-1907

13. Erejuwa O. O, Sulaiman S. A, and Ab Wahab M. S. Honey a novelantidiabetic agent. International Journal of Biological Sciences, 2012a; 8(6): 913-934

14. Rajabzadeh A, Saki GH, Hemadi M, Alli K. and Alli R. Evaluation of effect of honey and vitamin E on apoptosis in testes of rat exposed to noise stress. Jentashapir, 2012; 3(1169): 523-532.

15. Al-Waili N. Intrapulmonary administration of naturalhoney solution, hyperosmolar dextrose or hypoosmolar distill water to normal individuals and to patients with type- 2 diabetes mellitus or hypertension: their effects on blood glucose level, plasma insulin and C-peptide, blood pressure and peaked expiratory flow rate. European Journal of Medical Research, 2003; 8(7): 295-303.

16. Boyne A. F. and Ellman G. L. A methodology for analysis of tissue sulphydryl components. Anal. Biochem. 1972; 46(2): 639-653.

17. Reilly C. A. and Aust S. D. Measurement of lipid peroxidation. In: Bus J. S, et al. (Eds) Curr Protoc Toxicol. Academic Press, San Diego, 1999: 2.4.1-2.4.13 
18. Soodi M, Naghdia N. and Sharifzadeh M. Effect of lead. Iran J Pharm Res, 2008; 7(1): 43-51.

19. Erejuwa O. O, Sulaiman S. A, and Ab Wahab M. S. Fructose might contribute to the hypoglycemic effect of honey. Molecules, 2012b; 17(12): 1900-1915.

20. Sloboda D. M, Li M,Patel R, Clayton Z. E, Yap S. and Vickers M. H. Early life exposure to fructose and offspring phenotype: implications for long term metabolic homeostasis. J. Obes. 2014; 2014: 203474.

21. Ozkan H andKutlu T. The relationship of fructose consumption with MDA levels in rat liver and its effect on the expression levels of COX-2 and NRF-2 genes. Veteriner Fakultesi dergisi 2020. 